\title{
Ridurre il pregiudizio implicito in classe per favorire l'inclu- sione: un percorso di formazione con gli insegnanti pre-servizio
} Reducing implicit prejudice in classroom to promote inclusion: A training with preservice teachers Sara Gabrielli, Giordana Szpunar*, Stefano Livi***

\begin{abstract}
Riassunto
Il contesto scolastico rappresenta un luogo privilegiato per rispondere alle sfide della complessità del reale, promuovendo una metamorfosi del rapporto apprendimento-insegnamento (Morin, 2001). La gestione dei contesti educativi, caratterizzati per natura da molteplici differenze di cultura, abilità, genere e sensibilità (Dovigo, 2008), richiede lo sviluppo di un corpo di competenze che consentano all'insegnante di operare in una direzione interculturale e inclusiva.

Il contributo presenta l'articolazione e gli esiti di un percorso di formazione sulla riduzione del pregiudizio etnico rivolto a docenti pre-servizio iscritti al Corso di Laurea in Scienze della formazione primaria della Sapienza, Università di Roma. Tra gli obiettivi principali dell'intervento formativo, quello di ridurre il pregiudizio implicito ed esplicito degli studenti in una prospettiva più ampia di promozione delle loro competenze interculturali.
\end{abstract}

Parole chiave: riduzione del pregiudizio, insegnanti pre-service, empatia, competenze interculturali, inclusione.

\begin{abstract}
The school context can be considered as a privileged place where it is possible to answer to our time complexity, promoting a metamorphosis in the learningteaching relationship (Morin, 2001). Administrating multicultural classrooms requires different skills to allow teachers to promote interculturalism and inclusion, accounting the differences in terms of culture, competence, gender and sensitivity which characterize those contexts.
\end{abstract}

* Dottoranda in Psicologia sociale, dello sviluppo e ricerca educativa presso l'Università degli Studi di Roma "La Sapienza”. E-mail: sara.gabrielli@uniroma1.it.

** Professoressa associata di Pedagogia interculturale presso l'Università degli Studi di Roma “La Sapienza”. E-mail: giordana.szpunar@uniroma1.it.

*** Professore associato di Psicologia sociale presso l’Università degli Studi di Roma "La Sapienza”. E-mail: stefano.livi@uniromal.it. 
The paper shows setting and results of a training to reduce ethnic prejudice on preservice teachers in Sapienza, University of Rome. One of the aims was to reduce preservice teachers' implicit and explicit prejudice and, in a wider perspective, to promote their intercultural competences.

Keywords: prejudice reduction, pre-service teachers, empathy, intercultural competences, inclusion

\section{Introduzione}

L'affermazione di nuovi modelli interpretativi del mondo sociale e dell'idea di equità e di giustizia (cfr. per es. Sen, 2006; Nussbaum, 2011), il passaggio dal modello biomedico all'approccio biopsicosociale al funzionamento, alla disabilità e alla salute (OMS, 2004), la crescente complessità della composizione della popolazione scolastica, determinata dal progressivo aumento dei flussi migratori, hanno portato la riflessione pedagogica e educativa a spostarsi da un modello scolastico legato al concetto di integrazione a un modello orientato verso l'inclusione.

Per costruire contesti scolastici realmente inclusivi si rende necessaria la realizzazione di un processo di consapevole valorizzazione delle differenze. Valorizzare le differenze a scuola significa avere un'idea di differenza come risorsa, come «contributo alla comprensione della diversità» e come «possibile sostegno alla classe», e significa avere un'idea di classe come gruppo di lavoro in cui ognuno può collaborare a partire dalle proprie specificità (Dovigo, 2017).

La sfida posta dall'inclusione [...] implica dunque non semplicemente «fare posto» alle differenze - in nome di un astratto principio di tolleranza della diversità - ma piuttosto affermarle, metterle al centro dell'azione educativa in quanto nucleo generativo dei processi vitali che si sviluppano proprio attraverso lo scarto di prospettiva derivante dalle molteplici differenze di cultura, abilità, genere e sensibilità che attraversano il contesto scolastico (Dovigo, 2008, p. 17).

Una scuola inclusiva, oltre a favorire l'apprendimento e la partecipazione di tutti gli alunni, compresi quelli senza Bisogni Educativi Speciali, potrebbe rappresentare un fattore di protezione forte, proprio nella direzione della prevenzione di quegli stessi BES che originano o si aggravano a causa delle barriere scolastiche (Ianes, 2008).

Dal punto di vista delle differenze etniche, il processo di inclusione sembrerebbe ancora solo parzialmente realizzato nella scuola italiana, almeno stando 
ai dati relativi ai percorsi scolastici e formativi degli allievi con cittadinanza non italiana (ISMU, 2019; MIUR, 2019a). E questo nonostante la scuola italiana, in un'ottica decisamente inclusiva, si sia determinata da tempo nel percorrere una «via interculturale» (MPI, 2007).

La complessità della situazione appena descritta rende necessario ripensare la professione educativa, finora formata con un'attenzione prevalente ai contenuti, ridefinendo nuovi profili di competenze che forniscano all'insegnante la capacità di intervenire in modo efficace in contesti educativi caratterizzati dalla diversità.

In questo articolo si presenta un'esperienza di formazione con gli insegnanti pre-servizio iscritti al Corso di Laurea in Scienze della Formazione Primaria della Sapienza (Università di Roma). Il percorso di formazione prende ispirazione dal progetto realizzato con i bambini di alcune scuole primarie di Roma nell'anno scolastico 2018-2019 da un gruppo di ricerca dell'Università di Roma Tre (Gabrielli et alii, 2019). Il progetto Yesterday-Today-Tomorrow aveva come obiettivo principale quello di mettere a punto un insieme di attività educative per la riduzione del pregiudizio etnico, implicito ed esplicito. I risultati delle rilevazioni dei livelli di pregiudizio dei bambini sono stati positivi: le attività svolte in classe hanno ridotto il pregiudizio etnico dei bambini in modo significativo.

Le stesse attività sono state proposte agli insegnanti pre-servizio della Sapienza che avrebbero poi dovuto realizzarle nelle classi in cui stavano svolgendo il tirocinio ${ }^{1}$. Il percorso formativo è stato avviato con un duplice obiettivo: verificare l'ipotesi che le attività potessero avere un impatto positivo sulla riduzione del pregiudizio dei futuri insegnanti e offrire loro contenuti, strumenti e abilità per progettare e realizzare interventi educativi inclusivi nella scuola.

\section{L'insegnante come professionista interculturale}

L'Italia è un paese che può ormai decisamente definirsi multiculturale. Da decenni caratterizzata da flussi migratori più o meno importanti e, a seconda del momento storico-politico, provenienti da luoghi diversi, si confronta quotidianamente $\mathrm{e}$ in tutti $\mathrm{i}$ contesti sociali con la diversità culturale. La scuola è uno dei contesti sociali istituzionali maggiormente caratterizzato dalla presenza di persone con background migratorio e, per le persone migranti, rappresenta spesso il primo contesto di socializzazione del paese accogliente.

\footnotetext{
${ }^{1}$ La seconda parte del percorso formativo, vale a dire la realizzazione delle attività educative in classe, non si è potuta concludere a causa dell'emergenza sanitaria e della conseguente sospensione delle attività didattiche.
} 
Secondo l'ultimo rapporto MIUR (2019a) su Gli alunni con cittadinanza non italiana a.s. 2017/2018, con riferimento ai dati aggiornati al 31/08/2018, il $9,7 \%$ della popolazione scolastica ha un'origine migratoria (di prima e/o seconda generazione).

Se consideriamo solo la scuola dell'infanzia e la scuola primaria l'incidenza dei bambini con cittadinanza non italiana supera 1'11\%. In particolare, nella scuola dell'infanzia l'aumento dei bambini di origine migratoria è stato di sole 295 unità, ma continua a crescere la loro incidenza sul totale (da $10,7 \%$ a $11,1 \%)$ per effetto del calo di entità ben maggiore degli allievi italiani.

Nella scuola primaria, che rimane l'ordine che assorbe il maggior numero di studenti con cittadinanza non italiana, gli alunni stranieri sono circa 308.000, $1 ' 11,2 \%$ del totale.

Un dato che caratterizza l'evoluzione del fenomeno è che il $63 \%$ degli studenti con cittadinanza non italiana è nato in Italia. Nei diversi ordini di scuola, la proporzione dei nati in Italia sul totale degli studenti stranieri è dell' $84,4 \%$ nella scuola dell'infanzia, del $75,2 \%$ nella primaria.

I dati che meritano una particolare attenzione, in quanto rappresentano «una delle dimensioni di analisi attraverso cui valutare l'integrazione formativa e sociale degli studenti di origine migratoria» (MIUR, 2019, p. 50), sono quelli relativi alla regolarità dei percorsi scolastici (ritardi, abbandoni) e all'acquisizione delle competenze di base (ISMU, 2019). In generale il ritardo scolastico interessa il 30,7\% degli studenti con cittadinanza non italiana a fronte del 9,6\% degli studenti italiani. Il divario aumenta, prevedibilmente, all'aumentare dell' ordine scolastico: nella scuola primaria è in ritardo il $12,4 \%$ degli stranieri e 1' $1,7 \%$ degli italiani, nella secondaria di I grado è in ritardo il $32,4 \%$ degli stranieri e il 5,5\% degli italiani, nella secondaria di II grado è in ritardo il 58,2\% degli stranieri e il $20 \%$ degli italiani. Anche i dati sugli abbandoni presentano una situazione di svantaggio maggiore degli alunni con cittadinanza non italiana rispetto agli alunni italiani (MIUR, 2019b): la percentuale di stranieri che abbandona il percorso scolastico durante la secondaria di primo grado si attesta intorno al 3,3\% a fronte di una percentuale di 0,6 di studenti italiani; nel passaggio tra secondaria di primo grado e secondaria di secondo grado salgono le rispettive percentuali e sale anche il divario tra i due gruppi di studenti; nella secondaria di secondo grado la percentuale di alunni stranieri che abbandonano la scuola arriva all' $11,6 \%$ contro il 3,8\% degli italiani.

Per quel che riguarda le performance in termini di risultati scolastici e competenze di base, «gli alunni stranieri delle scuole secondarie di primo grado hanno mediamente mezzo punto in meno degli italiani nei voti di Italiano e Matematica» (ISTAT, 2016, p. 1) e hanno una percentuale di low performers, relativamente ai dati Invalsi 2016, più alta dei coetanei italiani in tutti gli ordini di scuola (ISMU, 2019). 
Il maggiore ritardo scolastico e il maggior tasso di abbandono precoce degli studi da parte degli alunni con background migratorio sono da attribuirsi, in parte, all'inserimento degli alunni in classi inferiori a quelle corrispondenti all'età anagrafica e alle non ammissioni e alle ripetenze. Anche l'aspetto linguistico ha certamente il suo peso. Lo dimostra il fatto che i dati appena citati assumono un andamento significativamente meno negativo se si prende in considerazione la sottocategoria degli studenti con CNI nati in Italia e il fatto che $i$ livelli di competenza in Matematica degli studenti con cittadinanza non italiana sono più vicini a quelli degli studenti italiani rispetto ai livelli in Italiano.

Tuttavia, non è da sottovalutare un ulteriore fattore che, con tutta probabilità, influenza la mancata inclusione degli alunni con cittadinanza non italiana. In generale, sappiamo che la dimensione emotiva condiziona in modo importante il processo di apprendimento (cfr. per es. Immordino-Yang, 2016) e a scuola è alimentata dalla percezione che lo studente ha del contesto scolastico e dal livello di benessere complessivo, determinato in larga misura dal clima di classe e dalla qualità delle relazioni tra studenti e tra studenti e docenti (Giovannini, Queirolo Palmas, 2002; Colombo, Santagati, 2014). Alcune ricerche hanno mostrato che il livello di benessere percepito è più basso per gli studenti stranieri che per gli studenti italiani (Stanzione, Szpunar, 2019). Ciò è da attribuire, in larga misura, ai meccanismi di marginalizzazione delle minoranze etniche (Rapari, 2007) e ai fenomeni della profezia che si autoavvera e della minaccia dello stereotipo, subiti dai membri di gruppi minoritari (Marini et alii, 2019), che spesso vengono minimizzati dai docenti (Serpieri, Grimaldi, 2013) e che incidono negativamente sulle performance scolastiche (cfr. per es. Schofield, 2006; Schmader et alii, 2008).

La lettura, seppur sommaria, dei dati rende evidente, come sottolinea bene Portera (2013, p. 124), «il fondamentale ruolo non solo della famiglia, ma soprattutto degli insegnanti, specie nel: riuscire ad assumere atteggiamenti di comprensione, accettazione, sostegno, stima e fiducia; non trasmettere stereotipi e pregiudizi; e soprattutto, saper gestire bene (senza rischio di xenofobia o di xenofilia) le differenze culturali degli alunni stranieri». Si rende necessario, dunque, che l'insegnante possegga alcune competenze che lo aiutino a gestire in modo efficace classi «a colori» (Ongini, Nosenghi, 2009) e a «dispensare un'educazione alla tolleranza e al rispetto delle differenze di ogni genere» (Perrenoud, 2002, p. 170).

Per essere in grado di progettare e realizzare interventi educativi orientati alla riduzione di pregiudizi e stereotipi, l'insegnante deve egli stesso saper riconoscere e ridurre i propri e quindi aver acquisito quella che Bennett (2002) definisce «sensibilità interculturale». D'altra parte, si tratta di un'esigenza messa in luce anche da recenti ricerche svolte in Italia, che sottolineano l'im- 
portanza dell'acquisizione delle competenze interculturali da parte dei professionisti dell'educazione (Onorati et alii, 2011; Reggio, Santerini, 2014). La ricerca e la letteratura sulle competenze interculturali è ormai ampia e articolata (cfr. per es. Deardoff, 2009). Uno degli aspetti più rilevanti che ci interessa mettere in luce, facendo riferimento anche alle indicazioni ministeriali sul tema (MPI, 2007; MIUR, 2014), è la necessità che l'insegnante sappia comunicare nel contesto educativo e progettare l'attività didattica e educativa tenendo conto delle differenze, promuovendo il rispetto, l'interazione, il dialogo, la riflessione, l'empatia, il decentramento, la sospensione del giudizio e riducendo pregiudizi, stereotipi, etnocentrismi. A tal fine, risulta di fondamentale importanza una formazione, pre-servizio e permanente, degli insegnanti orientata al potenziamento delle capacità di riconoscere le proprie cornici di riferimento e prenderne distanza, di assumere la prospettiva dell'altro e di comprenderne gli stati emotivi, di comprendere e valorizzare la diversità.

\section{II percorso di formazione degli insegnanti pre-servizio}

Con la finalità di sensibilizzare gli alunni della scuola primaria ai diritti fondamentali dell'uomo e contrastare la formazione del pregiudizio etnico, nel corso dell'anno scolastico 2018-2019 è stato proposto un progetto pilota (Gabrielli et alii, 2019). Tale progetto si è costituito come percorso educativo-didattico ed è stato realizzato in cinque classi quinte di scuola primaria nelle periferie di Roma, riconoscendo il ruolo fondamentale della scuola nello sviluppo o nella riduzione del pregiudizio etnico (Hello et alii, 2004; Baron, 2015; Bigler, Liben, 2006). Infatti, la scuola è uno dei contesti sociali più influenti sullo sviluppo dei bambini (Benard, 1995) e sulla promozione dell'autoefficacia, sull'autostima e sulle relazioni positive e rappresenta un luogo privilegiato in cui costruire la cittadinanza attiva (MPI, 2007; MIUR, 2012).

Tenendo conto delle strategie che la letteratura identifica efficaci nella riduzione del pregiudizio e nella promozione di pratiche antidiscriminatorie, il percorso educativo-didattico è stato suddiviso in otto incontri interdisciplinari basati su metodologie didattiche attive e partecipate, utilizzando il contatto diretto e indiretto, la promozione dell'empatia, l'assunzione di prospettiva (Hewstone, Voci, 2009; Pettigrew, Tropp, 2008; Batson et alii, 2002; Pica, Sciara, Livi e Pantaleo, 2017):

nella prima fase è stato focalizzato il vissuto personale degli studenti, indagando le loro conoscenze pregresse in merito alla migrazione; la seconda fase è stata incentrata sul contatto virtuale con i migranti, veicolato dall'utilizzo dei disegni realizzati da bambini migranti e dal confronto tra la propria esperienza e quella dei migranti; la terza fase 
ha previsto una serie di attività volte alla promozione dell'empatia, del perspective taking e della comprensione emotiva, chiedendo agli alunni di riscostruire esperienze di migrazione vicine alla loro realtà; l'ultima fase si è concentrata sull'identificazione nel processo di cambiamento dell'identità sperimentato dai migranti, grazie anche al contatto diretto con testimoni privilegiati (Gabrielli et alii, 2019, p.91).

Con l'obiettivo di attivare processi empatici e di riflessione, sono stati utilizzati disegni di bambini migranti della stessa età dei bambini - italiani e con cittadinanza non italiana - partecipanti al progetto nelle cinque scuole.

I disegni mostrati durante il percorso educativo-didattico fanno parte di un database visivo creato dall'associazione Yesterday, Today, Tomorrow, fondata dall'artista Bryan McCormack ${ }^{2}$. Tale database virtuale si costituisce di disegni raccolti nei centri di accoglienza del Mediterraneo, chiedendo ai migranti di differenti nazionalità e età di raccontare in forma grafica il loro passato, presente e futuro. Questa attività permette ai bambini partecipanti al progetto di riconoscere le voci dei migranti e promuovere un contatto indiretto e mediato dai disegni, facendo dialogare la loro esperienza con quella dei bambini migranti ospiti dei centri di accoglienza.

Il progetto pilota ha previsto anche la somministrazione di alcuni strumenti di rilevazione, pre e post intervento, del pregiudizio implicito (sottointeso e nascosto) ed esplicito (manifesto) - misurati rispettivamente attraverso il ChildIAT e un'intervista strutturata (Pirchio et alii, 2018) in gruppi sperimentali e di controllo.

I risultati mostrano una riduzione significativa del pregiudizio esplicito e implicito dopo l'intervento solo nei bambini delle classi sperimentali, e non in quelle di controllo (Perucchini et alii, 2019).

Tale progetto pilota sembra aver dimostrato che è possibile attuare interventi educativi-didattici che abbiano effetti sullo sviluppo del pregiudizio etnico nei bambini, in accordo con la letteratura che attribuisce il progresso di tale pregiudizio all'interazione tra fattori genetici e fattori esperienziali e sociali (Hatemi et alii, 2009). In particolare, l'incremento del pregiudizio nei bambini sembra essere condizionato dalle interazioni sociali infantili e dalle relazioni con gli adulti significativi (Pirchio et alii, 2018).

L'influenza che l'insegnante ha sullo sviluppo del pregiudizio etnico dei bambini, dunque, può essere rilevante. D'altra parte, in mancanza di «programmi formativi sul tema della diversità, gli insegnanti italiani sono poco consapevoli di quali strumenti sarebbero necessari per affrontare la multiculturalità» (Colombo, Santagati, 2014, p. 83). Inoltre, studi sulle credenze degli insegnanti pre-servizio (Hanushek et al., 2004; Catalano, Perucchini, 2016) eviden-

\footnotetext{
${ }^{2} \mathrm{Si}$ veda yttassociation.org.
} 
ziano i timori dei tirocinanti di non essere completamente preparati ad affrontare la realtà della classe, che diventa più complessa con la presenza di alunni con background migratorio. Infine, una formazione sui contenuti sembrerebbe non essere sufficiente per aumentare il senso di autoefficacia dei futuri insegnanti. In alcune ricerche sui corsi di formazione per insegnanti di sostegno emerge che «quanto più i corsisti approfondiscono la conoscenza di varie forme di disabilità (con particolare riferimento a quelle gravi), tanto più la loro fiducia nelle proprie capacità come futuri docenti non solo non aumenta, ma anzi diminuisce» (Dovigo, 2017, p. 99).

Considerando queste premesse, nell'a.a. 2019-2020 presso il Corso di Laurea in Scienze della formazione primaria della Sapienza è stata avviata una ricerca esplorativa sull'efficacia di un intervento formativo rivolto a studenti e a studentesse e orientato alla riduzione del pregiudizio etnico.

L'ipotesi di ricerca che ha guidato la formazione si è focalizzata su due livelli di intervento: da una parte, si è perseguito l'obiettivo di ridurre il pregiudizio che gli stessi futuri insegnanti potrebbero avere, anche implicitamente, nei confronti dei minori migranti (che rappresentano il 12,5\% della popolazione migrante nel mondo secondo i dati OECD del 2018), sensibilizzando al rispetto dei diritti umani. Alcuni studi hanno dimostrato quanto le aspettative e gli stereotipi degli insegnanti influiscano sui risultati didattici degli studenti, andando a consolidare il divario esistente tra studenti nativi e studenti con background migratorio (Paik, Walberg, 2007; Ferguson, 2003; Stiefel, Schwartz e Ellen, 2007; McKown, Weinstein, 2008). Infatti, gli stereotipi che si attivano nel momento in cui un insegnante categorizza un bambino come appartenente a un determinato gruppo etnico possono influenzarne il giudizio e il comportamento (Kawakami, Young e Dovidio, 2002). Sembra essere dimostrato che insegnanti con un pregiudizio etnico negativo siano portati a giudicare negativamente le prospettive accademiche degli studenti (Van den Bergh et alii, 2010).

Il secondo livello di intervento ha perseguito l'obiettivo di offrire ai partecipanti un insieme di conoscenze teoriche, un insieme di attività educative e un corpo di competenze utili per sviluppare la capacità di programmare e realizzare interventi didattici e educativi volti alla promozione di pratiche antidiscriminatorie e inclusive in classe.

Il gruppo di studenti che avrebbe frequentato il percorso di formazione è stato selezionato invitando tutti gli iscritti al III e IV anno di Corso di Laurea a chiedere ai propri tutor scolastici la disponibilità a realizzare nelle classi di riferimento le attività previste dal progetto pilota descritto sopra. Tutti i tirocinanti che hanno ricevuto una risposta positiva sono stati invitati a partecipare all'intervento formativo Educare all'empatia per favorire l'inclusione, che si è sviluppato in tre incontri di tre ore ciascuno, condotti nei mesi di novembre e dicembre 2019. Gli studenti che invece hanno ricevuto una risposta negativa 
dai propri tutor scolastici hanno partecipato a una serie di incontri formativi, con il tutor universitario, sul tema della progettazione didattica. Questo secondo gruppo ha costituito il gruppo di controllo della ricerca e gli studenti sono stati inseriti in una waiting list in modo che possano frequentare il percorso di formazione Educare all'empatia per favorire l'inclusione nell'a.a. 2020-2021.

Gli studenti di entrambi i gruppi, sperimentale e di controllo, hanno compilato pre e post intervento gli strumenti di rilevazione del pregiudizio implicito ed esplicito. In particolare, in questo articolo saranno presi in considerazione i dati relativi al pregiudizio implicito, che si attiva in modo automatico, è difficilmente controllabile e influenza soprattutto le interazioni non verbali tra persone. Il pregiudizio implicito è stato misurato attraverso l'Implicit Association Test (Greenwald et alii, 1998; Nosek et alii, 2007).

L'intervento formativo Educare all'empatia per favorire l'inclusione si è sviluppato nel modo che segue: nel primo incontro le partecipanti, tutte donne, hanno sperimentato una pratica laboratoriale, ripercorrendo tutte le attività proposte nel progetto pilota svolto con i bambini e descritto sopra, con l'obiettivo di far sperimentare - in forma di workshop - il percorso educativo-didattico per comprendere quali potrebbero essere le difficoltà incontrate dai bambini stessi. Le tirocinanti hanno, pertanto, disegnato il proprio passato, presente e futuro, condividendone i contenuti con il gruppo; hanno immaginato e visualizzato il passato, presente e futuro immaginando di essere migranti, sviluppando una riflessione di confronto tra la loro esperienza e quella migratoria. In questo modo si è favorito lo sviluppo dell'assunzione di prospettiva e dell'empatia, come strategie di riduzione del pregiudizio.

Successivamente sono stati mostrati i disegni di bambini e giovani adulti migranti, al fine di attivare i processi di immedesimazione e di assunzione di prospettiva e utilizzandoli come mediatori di contatto indiretto. In gruppi si è cercato di dare un volto agli autori dei disegni, immaginando quale potesse essere la loro storia e confrontando le ipotesi con la storia reale.

Il progetto per bambini prevedeva la somministrazione di un'intervista a casa, per indagare e scoprire la storia di migrazione familiare (anche all'interno dell'Italia stessa). Le tirocinanti hanno riflettuto in aula sulle loro esperienze personali e familiari, confrontandosi su quanto il movimento e la migrazione siano parte integrante della vita umana.

Il tema dell'identità e della sua costruzione è stato affrontato chiedendo alle partecipanti di individuare sei parole o brevi frasi per rispondere alla domanda "Chi sei tu?". In un secondo momento, sono state chiamate a selezionare e tenere solo due aspetti individuati. La riflessione si è sviluppata a partire da alcune domande guida: quali scelte guidano la selezione? Quali parti di identità siamo disposti a lasciare indietro? Quali devono abbandonare i migranti? 
Per ricostruire la riflessione e come debriefing, è stato chiesto alle studentesse di disegnare o elencare quali risorse inserirebbero in una valigia, immaginando di affrontare una migrazione.

L'incontro si è concluso raccontando nei dettagli il percorso educativo proposto nelle scuole e il progetto generale, sollecitando una riflessione su eventuali difficoltà incontrate e sull'anticipazione di quali difficoltà si potrebbero incontrare nella gestione delle attività in classe.

Durante il secondo incontro sono state prese in considerazione le principali teorie di riferimento sullo sviluppo e la riduzione del pregiudizio. Finalità dell'incontro è stata l'acquisizione di consapevolezza sui propri pregiudizi e stereotipi e di conoscenza di alcune delle strategie di riduzione del pregiudizio ritenute efficaci dalla letteratura di ambito psicosociale. Pregiudizi e stereotipi si sviluppano in parte inevitabilmente in ogni persona a causa dell'intrecciarsi di diversi fattori: il funzionamento cognitivo ed emotivo dell'individuo, l'influenza del gruppo sociale, le esperienze personali. Sui pregiudizi individuali è possibile, almeno in una certa misura, agire consapevolmente attivando l'attenzione in modo da ridurre il ricorso automatico agli stereotipi.

Questo controllo sul proprio comportamento può avere un effetto positivo sia sulla riduzione della trasmissione degli stereotipi condivisi sia sull'influenza delle aspettative dell'insegnante sui suoi studenti. Infatti, «le credenze, le intenzioni e gli atteggiamenti degli insegnanti possono giocare un ruolo più significativo del curriculum, della grandezza del gruppo classe e aspetti simili sul successo o sul fallimento individuale degli studenti» (Obidah, Teel, 2001, p. 107). Appare dunque fondamentale che gli insegnanti prendano consapevolezza di tali stereotipi, riconoscendone gli aspetti nascosti e non intenzionali. Si apre in tal modo la possibilità di sviluppare quelle che sono definite abilità autoregolatorie del pregiudizio (Pang e Park, 2002): anche insegnanti con basso pregiudizio sviluppano un meccanismo di autoregolazione quando riconoscono la presenza di una discrepanza tra la componente cognitiva e le risposte affettive alle differenze culturali (Pang, 2002).

Insieme alle partecipanti sono state ricostruite le definizioni dei termini "pregiudizio", "categorizzazione", "stereotipo", "discriminazione" (Pettigrew, Meertens, 1995; Bernardo, Vezzali e Giovannini, 2017). È stato preso in considerazione il processo di sviluppo del pregiudizio nei bambini e le principali strategie di contrasto e riduzione (cfr per es. Weber, Crocker, 1983; Allport, 1954; Brewer, Miller, 1984; Gaertner, Dovidio, 2000). Successivamente è stato chiesto alle studentesse di considerare in che modo tali strategie fossero state utilizzate nel progetto pilota mostrato loro.

Il terzo incontro ha previsto la presentazione di progetti utili alla riduzione del pregiudizio per bambini e bambine di scuola primaria. Nella seconda parte della lezione è stato chiesto alle partecipanti di lavorare in gruppi per progettare 
un'unità didattica di dieci ore da realizzare in classi di scuola primaria al fine di promuovere pratiche antidiscriminatorie e orientate alla riduzione del pregiudizio. L'attività laboratoriale ha riguardato le diverse forme di pregiudizio (etnico, di genere, nei confronti della disabilità) e i gruppi sono stati lasciati liberi di scegliere il nucleo su cui elaborare l'intervento e le relative strategie di riduzione del pregiudizio. Infatti, sebbene il progetto focalizzi soprattutto il pregiudizio etnico, è stato importante per le tirocinanti consolidare le conoscenze teoriche apprese sul concetto di pregiudizio e sulle strategie utili alla sua riduzione, estendendole ad altri ambiti di applicazione.

Il lavoro è stato poi completato a casa, sempre in gruppo, dalle tirocinanti e condiviso via e-mail con i diversi gruppi.

\section{Metodo}

\subsection{Campione}

Come detto, sono stati invitati a partecipare al progetto tutti gli studenti iscritti al III e IV anno del Corso di Laurea Scienze della Formazione Primaria. Al primo invio del link per la somministrazione degli strumenti hanno risposto 80 tirocinanti (3 maschi, 77 femmine), di cui 40 iscritti al III anno e 40 iscritti al IV anno. Di questi, 43 hanno confermato la partecipazione al progetto, compilando anche il questionario post-intervento.

Al fine di evitare che l'esperienza pregressa in ambito scolastico potesse risultare come fattore confondente rispetto agli obiettivi della ricerca, sono state prese in considerazione solo le risposte dei tirocinanti che non avessero precedente esperienza nelle scuole in nessun ruolo. Il campione così risultante è composto da 36 tirocinanti prive di esperienza nelle scuole, con età di 22,25 $(\mathrm{SD}=$ $2,68)$, tutte donne, delle quali 20 hanno partecipato al corso di formazione nei mesi di novembre e dicembre 2019, costituendo il gruppo sperimentale. Le restanti 16 hanno costituito il gruppo di controllo. 31 studentesse hanno affermato di frequentare le lezioni del corso di studi e 5 di essere non frequentanti; 18 sono iscritte al III anno di corso e 15 al IV anno.

\subsection{Procedura e strumenti}

I partecipanti sono stati invitati a compilare la versione online - tramite condivisione su InquisitWeb - dello IAT (Implicit Association Test) per misurare il pregiudizio etnico implicito (Greenwald et alii, 1998; Nosek et alii, 2007).

\footnotetext{
${ }^{3}$ Il progetto di ricerca ha previsto anche la somministrazione di strumenti standardizzati per
} 
Una settimana prima dell'intervento è stata proposta la somministrazione del pre-test e dalla settimana successiva all'intervento è stato proposto il posttest.

Il test è stato adattato alla lingua italiana e si basa sulla misura della forza delle associazioni automatiche (implicite) tra concetti, analizzando il tempo di latenza in un semplice compito (Greenwald, Nosek e Banaji, 2003). Lo strumento consiste di sette blocchi (Nosek et alii, 2005): il primo richiede di categorizzare fotografie di persone caucasiche, africane, asiatiche, indiane, arabe e sudamericane in due gruppi (Causasici e Non Caucasici) utilizzando il tasto I per la categoria disposta a destra e il testo E per la categoria disposta a sinistra. L'attribuzione delle categorie a destra o sinistra è casuale. Il secondo blocco richiede di categorizzare alcuni aggettivi (ad es. "meraviglioso", "stupendo", "tragico", "umiliante") in positivi e negativi, utilizzando sempre gli input della tastiera E e I.

Il terzo blocco combina fotografie e stimoli verbali, chiedendo sempre di categorizzare a destra o sinistra, in base alla posizione attribuita alle etichette Caucasici, Non caucasici, Positivi, Negativi. Questo blocco si configura come blocco di prova e allenamento, prima di procedere con il quarto blocco che replica il terzo, ma calcolando i tempi di risposta.

Nel quinto blocco la posizione delle etichette Caucasici e Non caucasici viene invertita e viene chiesto ai partecipanti di premere nuovamente i tasti $\mathrm{E} e$ I per categorizzare le fotografie.

I blocchi sei e sette sono analoghi ai blocchi tre e quattro, con le etichette invertite nel blocco cinque.

Durante tutto il test, non è possibile andare avanti finché fotografie e stimoli verbali non siano assegnati alla categoria corretta. In caso di errore appare una $\mathrm{X}$ rossa ed è sufficiente premere il tasto opposto della tastiera.

\section{Risultati}

Utilizzando il software SPSS (ver. 25) è stata condotta una ANOVA 2x2 con disegno misto, ponendo a fattore between la partecipazione al corso di formazione (vs. gruppo di controllo) e a fattore within i due tempi di somministrazione. Ipotizzando un effetto dell'età, è stata inserita tale variabile come covariata.

Tra gli effetti multivariati principali, come emerge nella tabella 1, appare una variazione significativa rispetto al tempo $\left(\mathrm{F}(1,33)=5.41, \mathrm{p}=.026, \eta_{\mathrm{p}}{ }^{2}=\right.$

la misurazione del pregiudizio esplicito e per la personalità, pre e post intervento, che non sono oggetto di questo articolo. 
.141) e si evidenzia un'interazione significativa $\left(F(1,33)=7.32, \mathrm{p}=.011, \eta_{\mathrm{p}}{ }^{2}=\right.$ .182) tra tempo della rilevazione e partecipazione al corso di formazione.

Si evidenzia, dunque, una variazione significativa nei livelli di pregiudizio implicito tra il pre ed il post-test e tale variazione sembra essere influenzata dalla partecipazione al progetto e all'intervento formativo. Quindi, possiamo affermare che le strategie considerate all'interno del percorso di formazione Educare all'empatia per favorire l'inclusione siano risultate efficaci per l'obiettivo di ridurre il pregiudizio implicito delle insegnanti pre-servizio.

Anche l'età sembra avere un'interazione significativa con la variabile within $\left(\mathrm{F}(1,33)=5.67, \mathrm{p}=.023, \eta_{\mathrm{p}}{ }^{2}=.147\right)$. In particolare, sembra che le partecipanti più giovani siano maggiormente ricettive all'intervento formativo - probabilmente per la minore esperienza in contesti educativi e scolastici, anche in vesti di tirocinanti - ottenendo un maggior decremento del loro livello di pregiudizio implicito e confermando l'ipotesi.

Tabella 1 - Effetti multivariati principali

\begin{tabular}{llll}
\hline & gl & F & Sign. \\
\hline Tempo & 1 & 5.41 & .026 \\
Tempo*Formazione & 1 & 7.32 & .023 \\
Tempo*Età & 1 & 5.67 & .011 \\
\hline
\end{tabular}

I confronti pairwise evidenziano, come mostrato nella tabella 2, l'andamento (grafico 1) decrescente del pregiudizio etnico nel gruppo sperimentale in modo significativo $(\mathrm{F}(1,33)=5.17, \mathrm{p}=.030)$ e non in quello di controllo, dove aumenta in maniera non significativa $(\mathrm{p}=.105)$.

Tabella 2 - Confronti pairwise

\begin{tabular}{llll}
\hline & gl & F & Sign. \\
\hline Sperimentale & 1 & 5.17 & .030 \\
Controllo & 1 & 2.78 & .105 \\
\hline
\end{tabular}

Come evidenziato nel grafico 1, nel gruppo sperimentale si osserva una riduzione del pregiudizio implicito tra la prima e la seconda somministrazione dello IAT che appare significativa e influenzata dalla partecipazione al percorso di formazione Educare all'empatia per favorire l'inclusione.

Lo stesso effetto non è, invece, rintracciabile nel gruppo di controllo, dove si osserva un incremento dei livelli di pregiudizio implicito, sebbene non sia significativo.

Si può affermare, dunque, che la partecipazione al corso Educare all'empatia per favorire l'inclusione ha ridotto in maniera significativa il pregiudizio implicito delle tirocinanti di Scienze della Formazione Primaria, confermando l'efficacia delle strategie scelte (empatia, contatto indiretto, perspective taking) 
e stimolando nelle insegnanti pre-servizio una riflessione sui propri pregiudizi e stereotipi.

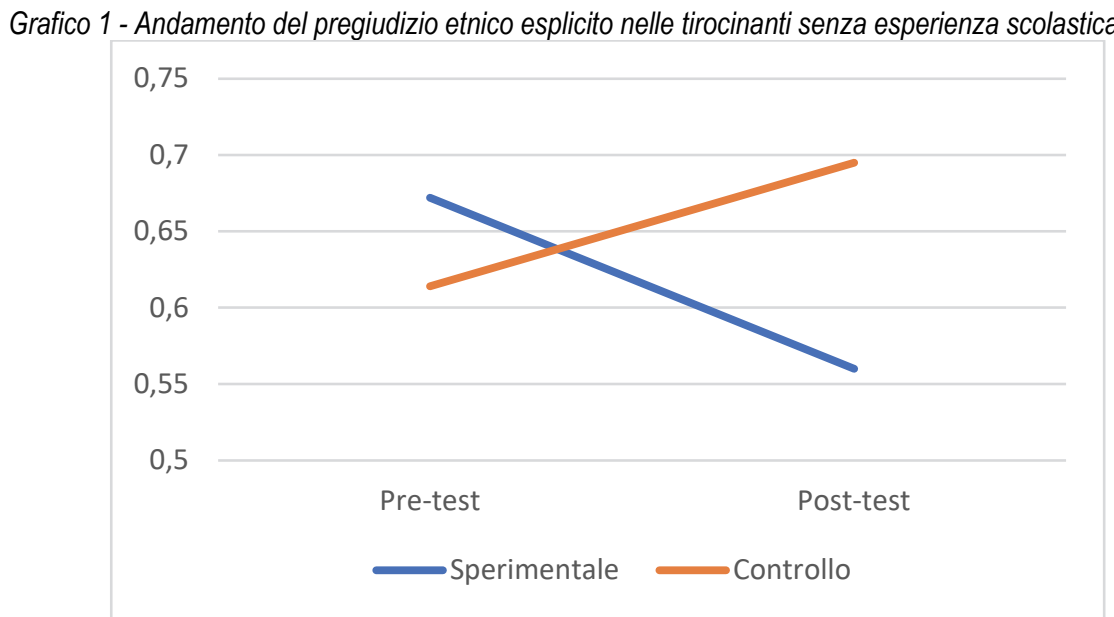

\section{Conclusioni}

Alcuni studi hanno indagato le credenze degli insegnanti pre-servizio relativamente all'inclusione di studenti con bisogni educativi speciali o con background migratorio (e.g. Crowson, Brandes, 2014; Garriott, Miller e Snyder, 2012), mentre ancora pochi studi ne indagano i pregiudizi (Pang, Park, 2013).

Essendo riconosciuta l'influenza del pregiudizio etnico implicito degli insegnanti sul pregiudizio etnico implicito degli studenti (Vezzali, Giovannini e Capozza, 2012), appare fondamentale volgere lo sguardo anche agli insegnanti pre-servizio che rappresentano il futuro del sistema scolastico.

Per questo motivo si è scelto di progettare un percorso formativo ancorato al tirocinio svolto all'interno del Corso di Laurea in Scienze della formazione primaria, con l'obiettivo di ridurre il pregiudizio etnico degli insegnanti preservizio e di sperimentare direttamente attività educative da poter riproporre anche nelle classi in cui insegneranno.

I risultati dell'analisi dei dati mostrano una riduzione significativa del pregiudizio implicito delle tirocinanti coinvolte nel corso di formazione e un andamento crescente, ma non significativo, del pregiudizio implicito delle tirocinanti nel gruppo di controllo. L'intervento descritto nel testo, dunque, appare 
efficace nel ridurre il pregiudizio implicito delle insegnanti pre-servizio, confermando la letteratura sul tema che identifica empatia, contatto indiretto e perspective taking come strategie efficaci.

Andrà valutato con un follow-up in una terza somministrazione se tale effetto resterà stabile e duraturo nel tempo.

La ricerca sul campione totale evidenzia che, purtroppo, negli insegnanti pre-servizio è presente un livello significativo di pregiudizio implicito, che potrebbe inconsapevolmente influenzare il futuro processo di insegnamento-apprendimento nelle classi in cui si troveranno ad insegnare. Si conferma, pertanto, la necessità di formare in modo adeguato i docenti alla gestione di classi multiculturali e di ridurre gli automatismi e gli stereotipi che ne determinano $i$ pregiudizi.

In prospettiva, andrà certamente implementata la ricerca, superando alcuni limiti attuali. In particolare, la selezione delle tirocinanti che hanno aderito al progetto si è svolta su base volontaria, non consentendo una randomizzazione del disegno di ricerca. Questa difficoltà ha portato ad una differenza iniziale nel livello di pregiudizio implicito nel gruppo di controllo e in quello sperimentale.

Al fine di ridurre tale differenza e ampliare il campione, la ricerca futura potrà essere inserita all'interno del tirocinio curriculare per tutti gli studenti $\mathrm{e}$ le studentesse del Corso di Laurea. Inoltre, potrebbe risultare interessante coinvolgere nel percorso formativo le docenti tutor già in servizio che accolgono le tirocinanti nelle loro classi. Il tirocinio indiretto svolto attraverso il corso di formazione potrebbe in questo modo legarsi al tirocinio diretto, chiedendo alle tirocinanti di realizzare nelle classi di tirocinio il percorso educativo-didattico già sperimentato nel progetto pilota (Gabrielli et alii, 2019; Perucchini et alii, 2019), misurando il pregiudizio implicito ed esplicito dei bambini e considerando eventuali relazioni tra il pregiudizio dei bambini e quello delle insegnanti pre-servizio.

\section{Bibliografia}

Baron A. S. (2015). Constraints on the development of implicit intergroup attitudes. Child Dev. Perspect, 9(1): 50-54. Doi: 10.1111/cdep.12105.

Batson C. D., Chang J., Orr R., and Rowland J. (2002). Empathy, Attitudes, and Action: Can Feeling for a Member of a Stigmatized Group Motivate One to Help the Group?. Personality and Social Psychology Bulletin, 28(12): 1656-1666. Doi: $10.1177 / 014616702237647$.

Benard B. (1995). Fostering resilience in children. Urbana: ERIC Clearinghouse on elementary and early childhood education. 
Bigler R. S., Liben L. S. (2006). A developmental intergroup theory of social stereotypes and prejudice. Advances in Child Development and Behavior, 34: 39-89. Doi. 10.1016/s0065-2407(06)80004-2.

Catalano M.G., Perucchini P. (2016). The use of a standardized instrument to promote reflective processes in pre-service teachers. Jure Conference, Helsinki.

Colombo M. e Santagati M. (2014). Nelle scuole plurali. Misure d'integrazione degli alunni stranieri. Milano: FrancoAngeli.

Crowson H.M., Joyce A.B. (2014). Predicting pre-service teachers' opposition to inclusion of students with disabilities: a path analytic study. Soc Psychol Educ, 17: 161-178. Doi: 10.1007/s11218-013-9238-2.

Deardorff D.K. (2009). The Sage Handbook of Intercultural Competence. Thousand Oaks: Sage.

Dovigo F. (2008). L'Index per l'inclusione: una proposta per lo sviluppo inclusivo della scuola. In: Booth T., Ainscow M., a cura di, L'Index per l'inclusione. Promuovere l'apprendimento e la partecipazione nella scuola. Trento: Edizioni Erickson.

Dovigo F. (2017). Pedagogia e didattica per realizzare l'inclusione. Guida all'Index. Roma: Carocci editore.

Ferguson R. F. (2003). Teachers' perceptions and expectations and the Black-White test score gap. Urban Education, 38: 460-507. Doi: 10.1177/0042085903038004006.

Gabrielli S., Szpunar G., Benvenuto G., Maricchiolo F., Catalano M.G., e Perucchini P. (2019). Riconoscere 1'Altro a Scuola. Il caso dei migranti. QTimes - Journal of Education, Technology and Social Studies, 3: 86-99. Doi: 11573/1337420.

Garriott P.P, Miller M. and Snyder L. (2003). Preservice Teachers' Beliefs about Inclusive Education: What Should Teacher Educators Know? Action in Teacher Education, 25(1): 48-54, doi: 10.1080/01626620.2003.10463292.

Giovannini G., Queirolo Palmas L., a cura di (2002). Una scuola in comune: esperienze scolastiche in contesti multietnici italiani. Torino: Fondazione Giovanni Agnelli.

Greenwald A. G., McGhee D. E., and Schwartz J. K. L. (1998). Measuring individual differences in implicit cognition: The Implicit Association Test. Journal of Personality and Social Psychology, 74: 1464-1480. Doi: 10.1037//0022-3514.74.6.1464.

Greenwald A. G., Nosek B. A., and Banaji M. R. (2003). Understanding and Using the Implicit Association Test: I. An Improved Scoring Algorithm. Journal of Personality and Social Psychology, 85: 197-216. Doi: 10.1037/0022-3514.85.2.197.

Hanushek E.A., Kain J.F., and Rivkin S.G. (2004). Why Public Schools Lose Teachers. J. Human Resources, XXXIX: 326-354. Doi: 10.3368/jhr.XXXIX.2.326.

Hatemi P. K., Funk C. L., Medlad S. E., Maes H. M., Silberg J. L., Martin N. G., and Eaves L.J. (2009). Genetic and environmental transmission of political attitudes over a lifetime. J. Polit., 3: 1141-1156. Doi: 10.1017/S0022381609090938.

Hello E., Scheepers P., Vermulst A., and Gerris J. R. M. (2004). Association between educational attainment and ethnic distance in young adults: socialization by schools or parents?. Acta Sociol., 47: 253-275. Doi: 10.1177/0001699304046222.

Hewstone M., Voci A. (2009). Diversità e integrazione: il ruolo del contatto intergruppi nei processi di riduzione del pregiudizio e risoluzione dei conflitti. Psicologia sociale, 1: 9-28. Doi: 10.1482/29208. 
Ianes D. (2008). L'Index per l'inclusione: dai Bisogni Educativi Speciali ai Livelli Essenziali di Qualità. In: Booth T., Ainscow M., a cura di, L'Index per l'inclusione. Promuovere l'apprendimento e la partecipazione nella scuola. Trento: Edizioni Erickson.

Immordino-Yang M. H. (2016). Emotions, Learning, and the Brain. Exploring the Educational Implications of Affective Neuroscience. New York: W.W. Norton \& Co.

ISMU (2019). Alunni con background migratorio in Italia. Emergenze e traguardi. https://www.ismu.org/wp-content/uploads/2018/10/1_2019-alunni-cn-background-migratorio.pdf.

ISTAT (2016). L'integrazione scolastica e sociale delle seconde generazioni. https://www4.istat.it/it/archivio/182866.

Kawakami K., Young H., and Dovidio J. F. (2002). Automatic stereotyping: Category, trait, and behavioral activations. Personality and Social Psychology Bulletin, 28: 315. Doi: 10.1177/0146167202281001.

Marini M., Liv S., Di Filippo G., Melchiori F.M., D’Ardia C., Benvenuto G. (2019). Aspetti individuali, interpersonali e sociali del bullismo etnico: studio su un campione nazionale di studenti della scuola secondaria di primo grado. ECPS Journal, 20: 103-120. Doi: 10.7358/ecps-2019-020-mari.

McKown C., Weinstein R. S. (2008). Teacher expectations, classroom context, and the achievement gap. Journal of School Psychology, 46: 235-261. Doi: 10.1016/j.jsp.2007.05.001.

MIUR (2012). Indicazioni nazionali per la Scuola dell'Infanzia e il primo ciclo di istruzione. http:/www.indicazioninazionali.it/wp-content/uploads/2018/08/decreto-ministeriale-254-del-16-novembre-2012-indicazioni-nazionali-curricolo-scuola-infanzia-e-primo-ciclo.pdf.

MIUR (2014). Linee guida per l'accoglienza e l'integrazione degli alunni stranieri. https://www.miur.gov.it/documents/20182/2223566/linee_guida_integrazione_alunni_stranieri.pdf/5e41fc48-3c68-2a17-ae751b5da6a55667?t=1564667201890.

MIUR (2019a), Gli alunni con cittadinanza non italiana a.s. 2017/2018, luglio 2019 (dati al 31/08/2018). https://miur.gov.it/documents/20182/250189/Notiziario+Stranieri+1718.pdf/78ab53c4-dd30-0c0f-7f40-bf22bbcedfa6?version $=1.1 \& \mathrm{t}=1562782116429$.

MIUR (2019b), La dispersione scolastica nell'a.s. 2016/2017 e nel passaggio all'a.s. 2017/2018, $\quad 2019$ luglio https://www.miur.gov.it/documents/20182/2155736/La+dispersione+scolastica+nell\%27a.s.201617+e+nel+passaggio+all\%27a.s.2017-18.pdf/1e374ddd-29ac-11e2-dede$4710 \mathrm{~d} 6613062$ ? version $=1.0 \& \mathrm{t}=1563371652741$.

Morin E. (2001). I sette saperi necessari all'educazione del futuro. Milano. Raffaello Cortina.

MPI (2007). La via italiana per la scuola interculturale e l'integrazione degli alunni stranieri.

Nosek B. A., Smyth F. L., Hansen J. J., Devos T., Lindner N. M., Ratliff (Ranganath) K. A., Smith C. T., Olson K. R., Chugh D., Greenwald A. G., and Banaji M. R. 
(2007). Pervasiveness and correlates of implicit attitudes and stereotypes. European Review of Social Psychology, 18: 36-88.

Nosek B., Greenwald A. G., and Banaji R. M. (2005). Understanding and using the Implicit Association Test: II. Method variables and construct validity. Pers. Soc. Psychol. Bull., 31: 166-180. Doi: 10.1177/0146167204271418.

Nussbaum M. (2011). Creating Capabilities: The Human Development Approach. London: Harvard University Press.

Obidah J.E., Manheim Teel K. (2001). Because of the Kids: Facing Racial and Cultural Differences in Schools. New York: Teachers College Press.

OECD (2018). The resilience of students with an immigrant background: Factors that shape well-being. Paris: OECD Publishing.

OMS (2004). ICF. Classificazione Internazionale del Funzionamento, della Disabilità e della Salute. Trento: Erickson.

Ongini V., Nosenghi C. (2009). Una classe a colori. Manuale per l'accoglienza e l'integrazione degli alunni stranieri. Milano: Antonio Vallardi Editore.

Onorati M.G., Bednarz F., e Comi G. (2011). Il professionista interculturale. Nuove competenze nella società del cambiamento. Roma: Carocci.

Paik S. J., Walberg H. J. (2007). Narrowing the achievement gap strategies for educating Latino, Black, and Asian students. New York: Springer.

Pang V.O. (2002). The courage to change: teacher prejudice review of because of the kids: facing racial and cultural differences in schools. Journal of Teacher Education, 53(5): 444-446. Doi: 10.1177/0022487102238071

Pang V. O., Park C. D. (2002). Examination of the selfregulation mechanism: Prejudice reduction in pre-service teachers. Paper presented at the annual meeting of the American Educational Research Association, New Orleans.

Pang V. O., Park C. D. (2003). Examination of the Self-Regulation Mechanism: Prejudice Reduction in Pre-Service Teachers. Action in Teacher Education, 25(3): 1-2, doi: 10.1080/01626620.2003.10734437.

Perrenoud P. (2002). Dieci nuove competenze per insegnare. Invito al viaggio [Dix nouvelles compétences pour einsegner, 1999]. Roma: Anicia.

Perucchini P., Gabrielli S., Maricchiolo F., Catalano M.G., e Szpunar G. (2020). Il progetto Yesterday-Today-Tomorrow: un percorso educativo per la riduzione del pregiudizio nella scuola primaria. In: Caldin, R., a cura di, Le società per la società: ricerca, scenari, emergenze: Vol II. Ricerca, scenari, emergenza sull'inclusione (226-233). Lecce: PensaMultimedia.

Pettigrew T. F., Tropp L. R. (2008). How does contact reduce prejudice? A meta analytic test of three mediators. European Journal of Social Psychology, 38: 922-934. Doi: $10.1002 /$ ejsp.504.

Pica G., Sciara S., Livi S., and Pantaleo G. (2017). Ethnic prejudice in person memory. Lessened retrieval-induced forgetting of negative traits ascribed to an AfricanAmerican target. Psicologia sociale, 12(3): 351-362. Doi: 10.1482/87890.

Pirchio S., Passiatore Y., Panno A., Maricchiolo F., and Carrus G. (2018). A Chip Off the Old Block: Parents' Subtle Ethnic Prejudice Predicts Children's Implicit Prejudice. Frontiers of psychology., 9: 110. Doi: 10.3389/fpsyg.2018.00110.

Portera A. (2013). Manuale di pedagogia interculturale. Roma-Bari: Laterza. 
Rapari S. (2007). L'osservazione delle relazioni multiculturali dei contesti scolastici. In: G. Maggioni, A. Vincenti, a cura di, Nella scuola multiculturale. Una ricerca sociologica in ambito educativo. Roma: Donzelli.

Reggio P., Santerini M., a cura di (2014). Le competenze interculturali nel lavoro educativo. Roma: Carocci.

Schmader T., Johns M., and Forbes C. (2008). An integrated process model of stereotype threat effects on performance. Psychological Review, 115(2): 336-356. Doi: 10.1037/0033-295X.115.2.336.

Schofield J. W. (2006). Migration Background, Minority, Achievement. Berlin: AKI.

Sen A. (2006). Scelta, benessere, equità. Bologna: il Mulino.

Serpieri R., Grimaldi E., a cura di (2013). Che razza di scuola. Praticare l'educazione interculturale. Milano: FrancoAngeli.

Stanzione I., Szpunar G. (2019). Fattori di benessere/disagio tra integrazione e inclusione scolastica degli studenti con cittadinanza non italiana nati all'estero. In: G. Benvenuto, P. Sposetti, e G. Szpunar, Tutti i bisogni educativi sono speciali. Riflessioni, ricerche, esperienze didattiche (pp. 117-130). Roma: Edizioni Nuova Cultura.

Stiefel L., Schwartz A. E., and Ellen I. G. (2007). Disentangling the racial test score gap: Probing the evidence in a large urban school district. Journal of Policy Analysis and Management, 26: 7-30. Doi: 10.1002/pam.20225.

Van den Bergh L., Denessen E., Hornstra L., Voeten M., and Holland R.W. (2010). The implicit attitudes of teachers: Relations to teacher expectations and the ethnic achievement gap. American Educational Research Journal, 47(2): 497-527. Doi: 10.3102/0002831209353594.

Vezzali L., Giovannini D. and Capozza D. (2012). Social antecedents of children's implicit prejudice: Direct contact, extended contact, explicit and implicit teachers' prejudice. European Journal of Developmental Psychology, 9(5): 569-581, doi: 10.1080/17405629.2011.631298. 